\title{
Fatty liver checkmates hepatitis B virus
}

\author{
Gayatri Ramakrishna ${ }^{1} \cdot$ Nirupma Trehanpati $^{1}$
}

Received: 24 August 2018/Accepted: 5 October 2018/Published online: 12 October 2018

(C) Asian Pacific Association for the Study of the Liver 2018

\section{Introduction}

In Asia-Pacific region, viral infections due to hepatitis $\mathrm{B}$ and $\mathrm{C}$ viruses are major etiological risk factors for liver disease and liver failure. However, a major concern now is the emergence of non-alcoholic fatty liver disease (NAFLD). A close association between the coexistence of NAFLD with liver diseases such as hepatitis $C$ virus, alcoholic liver disease and hemochromatosis is quite common. There are only few meta-analysis studies on the association between hepatitis B virus (HBV) and NAFLD and they are mostly these studies are from the Asia-Pacific region. Further, the fatty liver-HBV link as reported in epidemiological studies and in vitro cell culture studies are contradictory. To understand the HBV-fatty liver conundrum, a clinical animal model is of utmost importance. In this issue of Hepatology International, $\mathrm{Hu}$ et al. [1] have made use of the HBV-immunocompetent mouse model to demonstrate that hepatic steatosis inhibited HBV replication.

\section{Does fatty liver affect the $\mathrm{HBV}$ replication and seroclearance of HBVs antigen?}

Hepatitis B virus is hepatotrophic, and chronic infection can be life threatening due to cirrhosis, liver failure and hepatocellular carcinoma. Persistence or clearance of HBV depends on a number of factors such as the age of infection, genetic/epigenetic host factors and the immune status. About $1-2 \%$ of chronic HBV carriers may show spontaneous HBsAg seroclearance. Infact, few epidemiological studies have suggested that the two most important factors associated with HBV clearance are (a) age of disease onset

Gayatri Ramakrishna

rgayatri@ilbs.in; gayatrirama1@gmail.com

1 Department of Molecular and Cellular Medicine, Institute of Liver and Biliary Sciences (ILBS), D1 Block, Vasant Kunj, New Delhi 110070, India and (b) the presence of steatosis are [2-4]. A few of the meta-analysis studies have suggested an inverse correlation between HBV replication and steatosis; however, the mechanistic details remain still unanswered. Interestingly, chronic HBV patients with steatosis and high body mass index showed accelerated HBsAg seroclearance compared to those without fatty liver [5]. Further, the authors noted that HBsAg seroclearance occurred at younger age in patients with hepatic steatosis in comparison to those without steatosis. In yet another study, extremely obese male subjects showed significant HBsAg seroclearance, which was regardless of age [6]. As most of these reports are based on epidemiological evidences, it is important to confirm these associations using animal studies also to dissect out the underlying host mechanisms associated with viral clearance.

Interestingly, a previous study reported that $\mathrm{HBV}$ transgenic mice fed on high-fat diet showed reduced HBV replication, but with little inflammation and liver damage [7]. Now a report by $\mathrm{Hu}$ et al. [1], in the current issue of this journal, further supports the opposing relation between HBV replication and steatosis by using a non-transgenic immunocompetent mouse model.

$\mathrm{Hu}$ et al. [1] have developed a novel FVB/N mouse model of HBV persistence by hydrodynamically injecting HBV 1.3 replication plasmid in the tail vein. HBV replicated in the liver of these mice even after 14 weeks of injection in more than $70 \%$ animals. Using this HBV-persistent mouse model, the authors then tested if induction of fatty liver can interfere with HBV replication. On the 3rd day after HBV injection, the animals were divided into two groups: one group was fed a normal diet, while another group was fed a high-fat diet (HFD). Animals fed an HFD showed prominent hepatic steatosis by 14 weeks, while those on normal diet showed normal liver histopathology. However, mice fed on HFD showed a significant decline in the levels of HBV-DNA, HBeAg, HBsAg, HBcAg, and HBV pgRNA as compared to those fed a normal diet (Fig. 1). This indicates that HFD-induced steatosis caused inhibition of HBV replication. This new mouse model [1] exhibited an inflammatory response (higher levels of TNF 


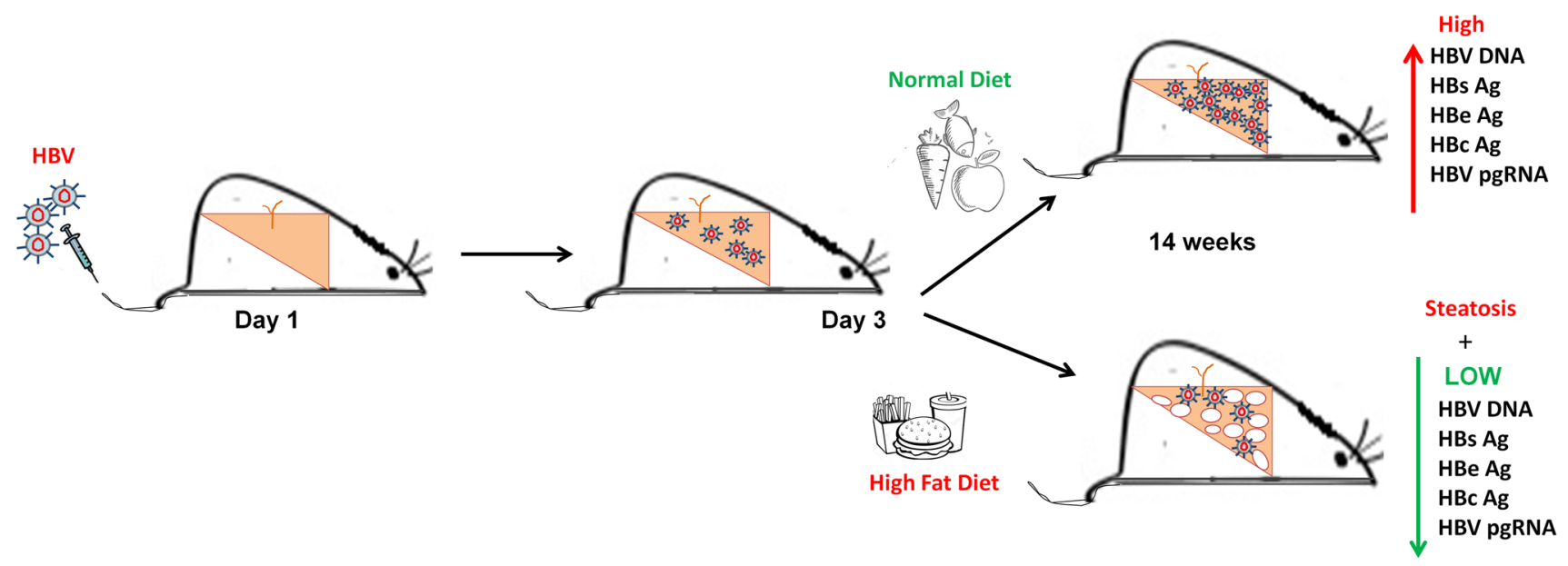

Fig. 1 HBV-NASH mouse model: High-fat diet induces steatosis and inhibits HBV replication in a non-transgenic immunocompetent mouse $(\mathrm{FVB} / \mathrm{N})$ model

$\alpha$, IL-1 $\beta$ and IL-6) and also liver damage (higher ALT and AST level), irrespective of the diet given to these animals. Both these studies concluded that low levels of HBV replication and HBs antigen levels in the animals fed a high-fat diet were not due to an overt immunological response, rather it seems to be dependent on a still unknown metabolic factor. However, a major drawback of the HBV-NASH rodent models is that the interpretations on non-involvement of immune response are based primarily on histopathology observations and levels of two or three inflammatory cytokines. A complete immune profiling may be necessary before drawing such a conclusion. Another major concern of the present study is the use of non-transgenic mice and non-permissive nature for HBV entry, which do not fully support the HBV replicative cycle. As a result, these animals may not efficiently produce cccDNA which is considered an important intermediate in HBV replication and life cycle. Further, in the present model, animals were first injected with HBV and later fed on HFD; however, it is equally important to test if HBV replication can still be prevented in a prior setting of fatty liver. Despite the limitations, the new HBV-NASH mouse model shows sustenance of HBV-DNA load even after 14 weeks of injection and thus seems to mimic a human condition of chronic HBV infection, which could be useful in future studies.

The HBV-NASH animal model does not provide the mechanistic details by which steatosis prevents HBV replication. It has been suggested previously that steatosis can either lead to cytoplasmic distribution of HBsAg or hepatocyte cell death, thereby leading to seroclearance of HBV [7]. It is now well known that steatosis is often associated with alterations in intestinal microbiota, leading to dysbiosis and inflammation. Recently it was reported that gut microbiota can activate liver immunity and HBV clearance [8]. It is quite possible that dysbiosis due to steatosis has a role in HBV clearance which needs to be validated. Ironically, on one hand, fatty liver limits HBV replication, on the other steatosis provides conducive environment for disease which can even culminate in liver cancer.

\section{Does HBV prevent steatosis?}

The HBV-NASH model described by $\mathrm{Hu}$ et al. [1] raises yet another pertinent question on the impact of HBV in the genesis of steatosis. In cell culture systems hepatitis $\mathrm{B}$ virus $\mathrm{X}$ protein is shown to favor lipid accumulation, in contrast the epidemiological studies indicate HBV as anti-steatogenic as it lowered the NAFLD risk [9-11]. A limitation in most of the meta-analysis studies is the use of ultrasound rather than biopsy-proven samples. In a study from India with $350 \mathrm{HBV}$ infected patients where liver biopsies were only available, one-third of the HBV chronic cases showed steatosis and also these patients had strong metabolic association with serum triglyceride levels [12]. Further, this study too indicated a negative correlation between HBV viral load and steatosis. Interestingly, HBsAg-positive subjects have much reduced levels of cholesterol compared to the HBsAg-negative group, thereby indirectly indicating a lipid-lowering ability of HBV [11]. However, proper caution needs to be taken while interpreting these results, because a lowered lipid profile in a setting of HBV could be impacted by many other factors, viz., the genetic/epigenetic background of the individual, diet, exercise regime and impact of drugs which were not considered in the 
study. Intriguingly, the HBV-NASH mice model developed by $\mathrm{Hu}$ et al. [1] and that of Zhang et al. [7] does not fully support the clinical observations of antisteatogenic effects of HBV. In fact, the HBV persistent mouse models fed on a high-fat diet showed development of liver steatosis as evidenced by histopathology and oil red staining. Further animal studies are needed to establish the mechanistic effect of HBV in preventing steatosis.

\section{Conclusions and future directions}

The study by $\mathrm{Hu}$ et al. [1] has elegantly proven that the development of fatty liver inhibited HBV replication in an immunocompetent mouse model of HBV replication. However, this study has not provided any mechanistic details of how and by what mechanism the fatty liver prevents the HBV life cycle. Intriguingly, this study points toward the role of host metabolic factors in inhibiting HBV replication. The existing epidemiological studies together with HBV-NASH animal models are suggestive of a paradigm shift regarding HBV seroclearance from an immune to a metabolic response, which needs to be evaluated further. Because of the non-permissive nature of non-transgenic mouse models of HBV, the humanized mouse models of HBV would be more appropriate to test the natural course of HBV replication in a steatotic background. None of the existing data on HBV-NASH association discuss the effect on the minichromosome formation (cccDNA) and this important aspect needs to be addressed in future studies. HBV clearance has been mainly reported in individuals with obesity and high BMI. However, lean NASH is an Asian entity where hardly any data are available on HBV replication or seroclearance.

In conclusion, the HBV-fatty liver conundrum needs a more comprehensive understanding of the mechanistic underlying cause, its clinical implications and the possible outcomes for antiviral treatment or response.

\section{Compliance with ethical standards}

Conflict of interest Gayatri Ramakrishna and Nirupma Trehanpati declare that they have no conflict of interests.

\section{References}

1. Hu D, Wang H, Wang H, Wang Y, Wan X, Yan W, et al. Nonalcoholic hepatic steatosis attenuates hepatitis B viral replication in an HBV-immunocompetent mouse model. Hepatol Int 2018. https://doi.org/10.1007/s12072-018-9877-7

2. Morales MR, Sendra C, Romero-Gomez M. Hepatitis B and NAFLD: lives crossed. Ann Hepatol 2017;16:185-187

3. Chu CM, Liaw YF. HBsAg seroclearance in asymptomatic carriers of high endemic areas: appreciably high rates during a longterm follow up. Hepatology 2007;45:1187-1192

4. Fung J, Yuen MF, Lai CL. The role of steatosis in HBsAg seroclearance for patients with chronic hepatitis B infection: fact or fiction? Dig Dis Sci 2013;58(1):20-22

5. Chu CM, Lin DY, Liaw YF. Clinical and virological characteristics post HBsAg seroclearance in hepatitis B virus carriers with hepatic steatosis versus those without. Dig Dis Sci 2013;58:275-281

6. Liu J, Yang HI, Lee MH, Lu SN, Jen CL, Wang LY, et al. Incidence and determinants of spontaneous hepatitis B surface antigen seroclearance: a community-based follow-up study. Gastroenterology 2010;139(2):474-482

7. Zhang Z, Pan Q, Duan XY, Liu Q, Mo GY, Rao GR, et al. Fatty liver reduces hepatitis $B$ virus replication in a genotype B hepatitis B virus transgenic mice model. J Gastroenterol Hepatol 2012;27(12):1858-1864

8. Chou HH, Chien WH, Wu LL, Cheng CH, Chung CH, Horng JH, et al. Age-related immune clearance of hepatitis B virus infection requires the establishment of gut microbiota. Proc Natl Acad Sci USA 2015;112(7):2175-2180

9. Kim KH, Shin HJ, Kim K, Choi HM, Rhee SH, Moon HB, et al. Hepatitis B virus $X$ protein induces hepatic steatosis via transcriptional activation of SREBP1 and PPARgamma. Gastroenterology 2007;132:1955-1967

10. Joo EJ, Chang Y, Yeom JS, Ryu S. Hepatitis B virus infection and decreased risk of nonalcoholic fatty liver disease: a cohort study. Hepatology 2017;65:828-835

11. Machado MV, Oliveira AG, Cortez-Pinto H. Hepatic steatosis in hepatitis B virus infected patients: meta-analysis of risk factors and comparison with hepatitis $\mathrm{C}$ infected patients. J Gastroenterol Hepatol 2011;26(9):1361-1367

12. Rastogi A, Sakhuja P, Kumar A, Hissar S, Jain A, Gondal R, et al. Steatosis in chronic hepatitis B: prevalence and correlation with biochemical, histologic, viral, and metabolic parameters. Indian J Pathol Microbiol 2011;54:454-459 